\title{
Tooth brushing for a longer and healthier life
}

\author{
E.E. van der Wall ${ }^{1}$
}

Published online: 8 February 2016

(C) The Author(s) 2016. This article is published with open access at Springerlink.com

Patients with coronary artery disease (CAD) who have no teeth have nearly double the risk of death compared with CAD patients with all of their teeth, according to research performed at the Uppsala Clinical Research Center, Uppsala, Sweden, and published in the European Journal of Preventive Cardiology in December 2015 [1].

The study of 15,456 patients from 39 countries found that levels of tooth loss were linearly associated with increasing rates of death and stroke. In recent decades, periodontal disease has emerged as a potential risk factor for CAD. Already in 1993, De Stefano et al. showed that dental disease was associated with an increased risk of CAD [2]. Similar to CAD, periodontal disease is a widespread disease with chronic inflammatory properties, influenced by numerous risk factors, of which lifestyle, poor oral health habits, age, smoking, diabetes and socioeconomic status are the most significant ones [3-10]. In patients with type 1 diabetes, periodontal disease is an independent predictor of long-term progression of calcium deposits in the coronary arteries [11]. Recently, genetic evidence for plasminogen as a shared genetic risk factor of $\mathrm{CAD}$ and periodontitis was shown [12]. Periodontal disease is the main cause of tooth loss and is related to poor dental hygiene, being one of the strongest risk factors for the occurrence of periodontal disease.

The study by Vedin et al. [1] is the first study to prospectively assess the relationship between tooth loss and outcomes in patients with CAD. The results are derived from a

E.E. van der Wall

eevanderwall@hotmail.com

Netherlands Society of Cardiology/Holland Heart House, Moreelsepark 1, 3511 EP Utrecht, The Netherlands substudy of the STABILITY trial [13, 14], which evaluated the effects of the lipoprotein-associated phospholipase A2 (Lp-PLA2) inhibitor darapladib versus placebo in patients with CAD. At the start of the study all patients completed a questionnaire about lifestyle factors, psychosocial factors, and their number of teeth in five different categories: (26-32 i.e. considered to have all teeth, 20-25, 15-19, 1-14 teeth and no teeth). Patients were followed for an average of 3.7 years. Associations between tooth loss and outcomes were calculated after adjusting for cardiovascular risk factors and socioeconomic status. The primary outcome was major cardiovascular events being a composite of cardiovascular death, myocardial infarction and stroke.

Patients who lost their teeth were older, smokers, female, less active and more likely to have diabetes, high blood pressure, more body fat and a lower level of education. During follow-up there were 1543 major cardiovascular events, 705 cardiovascular deaths, 1120 deaths from any cause and 301 strokes. After adjusting for cardiovascular risk factors and socioeconomic status, every increase in category of tooth loss was associated with a $6 \%$ increased risk of major cardiovascular events, $17 \%$ increased risk of cardiovascular death, $16 \%$ increased risk of all-cause death and $14 \%$ increased risk of stroke.

After adjusting for risk factors and socioeconomic status, the group with no teeth had a $27 \%$ increased risk of major cardiovascular events, $85 \%$ increased risk of cardiovascular death, $81 \%$ increased risk of all-cause death and $67 \%$ increased risk of stroke compared with those with all of their teeth.

The authors commented that the risk increase was linear with the highest risk in those with no remaining teeth. For example, the risks of cardiovascular death and all-cause death were almost double to those in patients with all teeth remaining. Coronary heart disease and periodontal disease 
share many risk factors such as smoking, unhealthy lifestyle and diabetes but after adjusting for these factors an apparently independent relationship between the two conditions was found. Many patients in the study had lost teeth: around $16 \%$ of patients had no teeth and about $40 \%$ were missing half of their teeth. Several mechanisms by which periodontal disease may impact CAD and cerebral strokes have been proposed, including detrimental effects on endothelial function, atherosclerosis progression and plaque stability $[15,16]$. Systemic inflammation generated by periodontal disease has been suggested as a possible causative pathway based on observations of increased levels of inflammatory markers in patients with periodontal disease [17]. The inflammation from periodontal disease is thought to trigger the atherosclerotic process and may explain the associations observed in the present study. However, the associations between periodontal disease and CAD included several cardiovascular outcomes and were independent of multiple risk factors. Therefore, the possibility of residual confounding or influence by unmeasured causal factors cannot be excluded.

During the study period 746 patients experienced a myocardial infarction. There was a numerically increased risk of myocardial infarctions for every increase in tooth loss. However, this finding was not significant after adjustment for risk factors and socioeconomic status. The occurrence of this phenomenon was not fully clear to the authors as they found strong associations with other cardiovascular outcomes including stroke.

To summarise, in this large global cohort of patients with $\mathrm{CAD}$, self-reported tooth loss predicted adverse cardiovascular outcomes and all-cause death independent of cardiovascular risk factors and socioeconomic status [1]. Every increased level of tooth loss was associated with a $6 \%$ increased risk of major cardiovascular events, and an approximately $15 \%$ higher risk of cardiovascular death, death from any cause and stroke. Overall, CAD patients with no teeth had nearly twice the risk of death to those patients with all of their teeth. The relationship between tooth loss and outcomes in CAD patients has not previously been evaluated in a prospective study. However, it is too premature to conclude that periodontal disease directly causes adverse events in CAD patients. According to the authors, tooth loss remains an easy and inexpensive way to identify patients at higher risk who need more intense dental instructions as poor dental hygiene remains one of the strongest risk factors for periodontal disease. As a result, one should intensively hang onto tooth brushing and flossing in order to live longer and healthier!

Open Access This article is distributed under the terms of the Creative Commons Attribution 4.0 International License (http://creativecommons.org/licenses/by/4.0/), which permits unrestricted use, distribution, and reproduction in any medium, provided you give appropriate credit to the original author(s) and the source, provide a link to the Creative Commons license, and indicate if changes were made.

\section{References}

1. Vedin O, Hagström E, Budaj A, On behalf of the STABILITY Investigators, et al. Tooth loss is independently associated with poor outcomes in stable coronary heart disease. Eur J Prevent Cardiol. 2015; doi:10.1177/2047487315621978.

2. DeStefano F, Anda RF, Kahn HS, Williamson DF, Russell CM. Dental disease and risk of coronary heart disease and mortality. BMJ. 1993;306:688-91.

3. Janket SJ, Baird AE, Chuang SK, et al. Meta-analysis of periodontal disease and risk of coronary heart disease and stroke. Oral Surg Oral Med Oral Pathol Oral Radiol Endod. 2003;95:559-69.

4. Bahekar AA, Singh S, Saha S, et al. The prevalence and incidence of coronary heart disease is significantly increased in periodontitis: a meta-analysis. Am Heart J. 2007;154:830-7.

5. Schenkein HA, Loos BG. Inflammatory mechanisms linking periodontal diseases to cardiovascular diseases. J Periodontol. 2013;84:S51-69. doi:10.1902/jop.2013.134006.

6. Smit RA, Trompet S, de Craen AJ, Jukema JW. Using genetic variation for establishing causality of cardiovascular risk factors: overcoming confounding and reverse causality. Neth Heart J. 2014;22:186-9. doi:10.1007/s12471-014-0534-z.

7. Leening MJ, Siregar S, Vaartjes I, et al. Heart disease in the Netherlands: a quantitative update. Neth Heart J. 2014;22:3-10. doi:10.1007/s12471-013-0504-x.

8. Snaterse M, Scholte Op Reimer WJ, Dobber J, et al. Smoking cessation after an acute coronary syndrome: immediate quitters are successful quitters. Neth Heart J. 2015;23:600-7. doi:10.1007/ s12471-015-0755-9.

9. Krenning BJ, Van der Heiden K. Should ethnicity be included in cardiovascular risk stratification? Neth Heart J. 2015;23:42-3. doi:10.1007/s12471-014-0634-9.

10. Van der Wall EE. Lifestyle for a lifetime. Neth Heart J. 2015;23:559-62. doi:10.1007/s12471-015-0764-8.

11. Groves DW, Krantz MJ, Hokanson JE, et al. Comparison of frequency and duration of periodontal disease with progression of coronary artery calcium in patients with and without type 1 diabetes mellitus. Am J Cardiol. 2015;116:833-7. doi:10.1016/j. amjcard.2015.06.006.

12. Schaefer AS, Bochenek G, Jochens A, et al. Genetic evidence for PLASMINOGEN as a shared genetic risk factor of coronary artery disease and periodontitis. Circ Cardiovasc Genet. 2015;8:159-67. doi:10.1161/CIRCGENETICS.114.000554.

13. White HD, Held C, Stewart R, Tarka E, STABILITY Investigators, et al. Darapladib for preventing ischemic events in stable coronary heart disease. N Engl J Med. 2014;370:1702-11. doi:10.1056/ NEJMoa1315878.

14. Vedin O, Hagström E, Gallup D, et al. Periodontal disease in patients with chronic coronary heart disease: prevalence and association with cardiovascular risk factors. Eur J Prev Cardiol. 2015;22:771-8.

15. Johansson CS, Ravald N, Pagonis C, Richter A. Periodontitis in patients with coronary artery disease: an 8-year follow-up. J Periodontol. 2014;85:417-25. doi:10.1902/jop.2013.120730.

16. Van Opstal JM, de Weerd GJ. Bad teeth and strange behaviour. Neth Heart J. 2006;14:66-7.

17. De Boer SP, Cheng JM, Rangé H, et al. Antibodies to periodontal pathogens are associated with coronary plaque remodeling but not with vulnerability or burden. Atherosclerosis. 2014;237:84-91. doi:10.1016/j.atherosclerosis.2014.08.050. 